\title{
Diagnostic error in hospitals: finding forests not just the big trees
}

Laura Zwaan (D) , ${ }^{1}$ Hardeep Singh (i) ${ }^{2}$

${ }^{1}$ Institute of Medical Education Research Rotterdam, Erasmus Medical Center, Rotterdam, The Netherlands

${ }^{2}$ Center for Innovations in Quality, Effectiveness and Safety, Michael E DeBakey Veterans Affairs Medical Center and Baylor College of Medicine, Houston, Texas, USA

\section{Correspondence to}

Dr Laura Zwaan, Institute of Medical Education Research Rotterdam, Erasmus Medical Center, Rotterdam 3015 CN, The Netherlands;

l.zwaan@erasmusmc.n

Accepted 22 June 2020 Published Online First 4 August 2020

\section{Linked}

- http://dx.doi.org/10.1136/ bmjgs-2020-010896

- http://dx.doi.org/10.1136/ bmjqs-2019-010822

\section{Check for updates}

(C) Author(s) (or their employer(s)) 2020. No commercial re-use. See rights and permissions. Published by BMJ.

To cite: Zwaan $\mathrm{L}$, Singh $\mathrm{H}$. BMJ Qual Saf

2020:29:961-964.
Diagnostic errors in hospital medicine have mostly remained in uncharted waters. ${ }^{1}$ This is partly because several factors make measurement of diagnostic errors challenging. Patients are often admitted to hospitals with a tentative diagnosis and need additional diagnostic investigations to determine next steps. This evolving nature of a diagnosis makes it hard to determine when the correct diagnosis could have been established and if a more specific diagnosis was needed to start the right treatment. ${ }^{2}$ Hospitalised patients also may have diagnoses that are atypical or rare and pose dilemmas for treating clinicians. As a result, delays in diagnosis may not necessarily be related to a diagnostic error. Furthermore, what types of diagnostic errors occur in the hospital and their prevalence depends on how one defines them. Different approaches to define them have included counting missed, wrong or delayed diagnoses regardless of whether there was a process error; ${ }^{3}$ counting them only when there was a clear 'missed opportunity' ie, something different could have been done to make the correct or timely diagnosis $;{ }^{4}$ or diagnostic adverse events (ie, diagnostic errors resulting in harm); ${ }^{5}$ all leading to views of the problem through different lenses.

Two articles in this issue of the journal provide new insights into the epidemiology of diagnostic errors in hospitalised patients. ${ }^{6} 7$ Gunderson and colleagues conducted a systematic review to determine the prevalence of harmful diagnostic errors in hospitalised patients. ${ }^{6}$ Raffel and colleagues studied readmitted patients using established methods for diagnostic error detection and analysis to gain insights into contributing factors. ${ }^{7}$ Both studies advance the science of measurement and understanding of how to reduce diagnostic error in hospitals. We discuss the significance of the results for hospital medicine and implications for emerging research and practice improvement efforts.

\section{FINDING DIAGNOSTIC ERRORS IN HOSPITALS}

Gunderson and colleagues performed a systematic review and meta-analysis to inform a new estimate for the prevalence of diagnostic adverse events among hospitalised patients, a rate of $0.7 \% .^{6}$ Their review shows how diagnostic error is a global problem, with studies from countries across five continents. The prevalence however is lower than what might be expected looking at previous research, mostly in outpatient care, and based on expert estimates. ${ }^{8-11}$ The prevalence of diagnostic error in hospital care may be lower because outpatient care, especially primary care, has the challenging task of identifying patients with a serious disease from a large sample of patients who present with common symptoms and mostly benign non-urgent diseases. A higher state of attention in the hospital and higher prior probability of a patient having a more serious disease may also reduce the likelihood of something being missed (ie, the prevalence effect). ${ }^{12} 13$ Furthermore, the hospital setting offers more diagnostic evaluation possibilities (consultations, imaging, laboratory) and more members of the diagnostic team to alert a clinician on the wrong diagnostic track.

The heterogeneity of the studies in the review and meta-analysis and a broad scope may also explain the lower prevalence rate. ${ }^{614}$ The included studies did not have an exclusive focus on detecting diagnostic errors but rather aimed to identify all types of adverse events, including medication and surgical adverse events, ${ }^{515}$ which are relatively easier to measure. Consequently, the data collection instruments were likely not sufficiently sensitive 
to pick up diagnostic adverse events, resulting in an underestimation. Some diagnostic adverse events may also be classified as 'other' types; for instance delayed diagnosis of a wound leakage after surgery is often considered a surgical complication and not categorised as a delay in diagnosis. ${ }^{16}$ Studies in the review also detected adverse events (ie, errors that resulted in harm) ${ }^{6}$ which is a subgroup of diagnostic errors, because not every diagnostic error results in harm. ${ }^{17}$ Lastly, while the random selection of patients is a strength for determining prevalence of medical error, not all admissions involve making a diagnosis-patients are often hospitalised for treatment and procedures. As the literature in the area becomes more robust, future reviews may be able to provide an updated estimate. For now, Gunderson and colleagues estimate 250,000 diagnostic adverse events occur annually in the USA, which should be alarming enough to warrant attention and intervention.

While the study by Raffel and colleagues is not a true prevalence study (it only evaluated 7-day readmissions), it uses dedicated tools to identify diagnostic error in hospitals, a crucial next step. By examining a subset of hospital admissions at greater risk of diagnosis-related problems (ie, readmissions within 7 days after hospital discharge) and by using tools dedicated to identifying diagnostic error, the investigators were able to describe error types and contributing factors. The advantage of studying such a high-risk sample is that diagnostic errors can be found more efficiently, that is, the positive predictive value is higher than if you review all consecutive patients. This could identify a higher number of cases to identify contributing factors. While the positive predictive value they achieved through this method was still rather low, methods to selectively identify diagnostic errors are valuable in measurement efforts. Future studies could build on this work to develop sampling methods with higher predictive values that can be used by others for research and practice improvement.

\section{DISEASES AT RISK FOR DIAGNOSTIC ERROR IN THE HOSPITAL SETTING}

Types of conditions involved in diagnostic error in both studies reflect a broad range of diseases commonly identified in previous studies, such as malignancies, pulmonary embolism, aortic aneurysm and infections. ${ }^{5818}$ A recent malpractice claims-based study has led some to suggest that initial diagnostic error reduction efforts, including allocation of funding for research and quality measurement/improvement, should focus on three broad types of disease categories, the so-called 'Big Three', namely cancer, infections and cardiovascular diseases, because they are highly prevalent and result in significant harm. ${ }^{11} 1920$ These three disease categories cover a large portion of diagnoses made in medicine. Indeed, data beyond claims also suggest that diagnostic errors in each of these categories are common. ${ }^{518}$ However, diagnostic errors span a large range of other diseases as shown in both studies, which is similar to what prior studies have found. For instance, in one primary care study, 68 unique diagnoses were missed with the most common condition accounting for only $6.7 \%$ of errors. ${ }^{21}$

\section{CONTRIBUTING FACTORS IN HOSPITAL MEDICINE}

Raffel and colleagues applied established tools (ie, SAFER $\mathrm{Dx}^{22}$ and $\mathrm{DEER}^{23}$ ) to identify contributing factors. They found that most of these involved failures in clinical assessment and/or testing. Contributing factors in these two domains occurred in more than $90 \%$ of diagnostic errors, a high proportion consistent with previous work. ${ }^{8}{ }^{17} 18$ Furthermore, these main contributing factors are common across diagnostic errors regardless of the diseases involved. For instance, similar process breakdowns emerge across different types of missed cancer diagnoses. ${ }^{24-26}$

\section{FINDING 'FORESTS' NOT JUST THE 'BIG TREES' TO ENABLE SCIENTIFIC PROGRESS}

So should initial scientific efforts just target disease categories? And if so, should they address just the 'Big Three'? Data from prior studies across different settings, including those from Gunderson and Raffel and colleagues, find large diversity in misdiagnosed diseases. ${ }^{5-7182127}$ This suggests that an exclusive focus on the 'Big Three' would neglect a substantial proportion of other common and harmful diagnostic errors. ${ }^{27}$ Furthermore, research on contributing factors of diagnostic errors reveals a number of common system and process factors that would require robust diseaseagnostic approaches. If funding and advocacy for diagnostic safety becomes mostly disease oriented, it will pull resources away from broader 'disease-agnostic' research and quality improvement efforts needed to understand and address these underlying system and process factors. ${ }^{28}$ Biomedical research is already quite disease focused and supported by many disease-specific institutes and this now needs to be balanced by work that catalyses much-needed foundational and crosscutting healthcare delivery system improvements.

We would thus recommend a balanced strategy that carefully combines disease-specific and diseaseagnostic approaches to help address common contributing factors, system issues and process breakdowns for diagnostic error that cut across these many unique diseases. For example, if new quality measures to quantify delays in colorectal cancer diagnosis and missed diagnosis of sepsis are developed, we would also need 'disease-agnostic' studies that evaluate the implementation and effectiveness of such measures. This includes how they fit within current measurement programmes, what their measurement burden is and what the unintended consequences may be. A combined approach would create more synergistic and collaborative understanding in addition to enabling 
application of common frameworks and approaches to multiple conditions, rather than 'reinventing the wheel' for each disease or disease category. This type of approach may have a larger population-based impact and help us see the entire 'forest' to reduce diagnostic error.

\section{IMPLICATIONS FOR PRACTICE IMPROVEMENT}

A crucial first step for improving diagnosis in hospitals is to create programmes to identify and analyse diagnostic errors. ${ }^{29}$ Most hospitals have systems and programmes in place to report and analyse safety issues such as falls, surgical complications and medication errors, but they do not capture diagnostic errors. With increased recognition of risks for diagnostic error, hospitals should use recent guidance, such as from the US Agency for Healthcare Research and Quality, and consider pragmatic measurement approaches to start identifying and learning from diagnostic errors. ${ }^{30}$

To reduce cognitive errors, 'cognitive debiasing strategies' have been widely recommended. ${ }^{31}$ However, there is increasing evidence that those strategies are not effective for diagnostic error reduction and recent insights have revealed lack of knowledge as the fundamental cause of errors in the diagnostic reasoning process. ${ }^{32-34}$ Next steps for practice improvement would therefore need to involve studying the role of knowledge and its interplay with cognitive processes. Interventions should explore opportunities to increase clinicians' knowledge base (eg, by education and feedback) as well as testing and implementing clinical decision support systems to allow for timely access to the relevant knowledge. While specific interventions need more development and testing, other general safety practices such as better collaboration with the laboratory and radiology departments to facilitate more accurate ordering and interpretation of the tests, ${ }^{33}$ are ready for adoption.

\section{CONCLUSIONS}

Two studies ${ }^{67}$ of diagnostic error in hospital medicine-by Gunderson and colleagues and Raffel and colleagues-have advanced our knowledge about its epidemiology. Consistent with prior studies, a large range of diseases and a whole host of common contributory factors are involved. Although the estimated prevalence of diagnostic error relies on data from prior studies conducted during an era of limited dedicated tools to identify diagnostic errors, these numbers have significant research and practice implications. Measurement science is still evolving but both studies should inspire all hospitals to apply more contemporary methods to identify and analyse diagnostic errors for learning and improvement. Given that errors across multiple diseases in multitude of settings have many common contributing factors, disease-agnostic approaches focused on common systems and process contributory factors are likely to have significant benefit and should be emphasised in further research and development efforts.

Twitter Laura Zwaan@laurazwaan81 and Hardeep Singh @HardeepSinghMD

Contributors Both authors drafted the editorial and approved of the final version.

Funding LZ is in part supported by a VENI grant from the Dutch National Scientific Organization (NWO; 45116032). HS is funded in part by the Houston Veterans Administration (VA) Health Services Research and Development (HSR\&D) Center for Innovations in Quality, Effectiveness, and Safety (CIN13-413), the VA HSR\&D Service (CRE17-127 and the Presidential Early Career Award for Scientists and Engineers USA 14-274), the VA National Center for Patient Safety, the Agency for Healthcare Research and Quality (R01HS27363), the CanTest Research Collaborative funded by a Cancer Research UK Population Research Catalyst award (C8640/ A23385) and the Gordon and Betty Moore Foundation.

Competing interests None declared.

Patient consent for publication Not required.

Provenance and peer review Commissioned; internally peer reviewed.

\section{ORCID iDs}

Laura Zwaan http://orcid.org/0000-0003-3940-1699

Hardeep Singh http://orcid.org/0000-0002-4419-8974

\section{REFERENCES}

1 Singh H, Graber ML. Improving Diagnosis in Health Care--The Next Imperative for Patient Safety. N Engl J Med 2015;373:2493-5.

2 Zwaan L, Singh $\mathrm{H}$. The challenges in defining and measuring diagnostic error. Diagnosis 2015;2:97-103.

3 Graber ML, Franklin N, Gordon R. Diagnostic error in internal medicine. Arch Intern Med 2005;165:1493-9.

4 Singh H. Editorial: helping health care organizations to define diagnostic errors as missed opportunities in diagnosis. Jt Comm J Qual Patient Saf 2014;40:99-AP1.

5 Zwaan L, de Bruijne M, Wagner C, et al. Patient record review of the incidence, consequences, and causes of diagnostic adverse events. Arch Intern Med 2010;170:1015-21.

6 Gunderson CG, Bilan VP, Holleck JL, et al. Prevalence of harmful diagnostic errors in hospitalised adults: a systematic review and meta-analysis. BMJ Qual Saf 2020;29:1008-18.

7 Raffel KE, Kantor MA, Barish P, et al. Prevalence and characterization of diagnostic error among 7-day all-cause Hospital medicine readmissions: a retrospective cohort study. BMJ Qual Saf 2020;29:971-9.

8 Singh H, Meyer AND, Thomas EJ. The frequency of diagnostic errors in outpatient care: estimations from three large observational studies involving us adult populations. BMJ Qual Saf 2014;23:727-31.

9 Schwartz A, Elstein AS. Clinical reasoning in medicine. In: Clinical Reasoning in the health professions. 3rd edn. Edinburgh: Elsevier, 2008: 223-34.

10 Berner ES, Graber ML. Overconfidence as a cause of diagnostic error in medicine. Am J Med 2008;121:S2-23.

11 Newman-Toker DE, Wang Z, Zhu Y, et al. Rate of diagnostic errors and serious misdiagnosis-related harms for major vascular events, infections, and cancers: toward a national incidence estimate using the "Big Three". Diagnosis 2020. 
12 Wolfe JM, Horowitz TS, Kenner NM. Cognitive psychology: rare items often missed in visual searches. Nature 2005;435:439-40.

13 Evans KK, Birdwell RL, Wolfe JM. If you don't find it often, you often don't find it: why some cancers are missed in breast cancer screening. PLoS One 2013;8:e64366.

14 Shojania KG, Marang-van de Mheen PJ, de MPJM. Identifying adverse events: reflections on an imperfect gold standard after 20 years of patient safety research. BMJ Qual Saf 2020;29:265-70.

15 Desapriya EBR, Baker GR, Norton PG. Canadian adverse events study. CMAJ 2004;171:834-5.

16 Zegers M, de Bruijne MC, de Keizer B, et al. The incidence, root-causes, and outcomes of adverse events in surgical units: implication for potential prevention strategies. Patient Saf Surg 2011;5:13.

17 Zwaan L, Thijs A, Wagner C, et al. Relating faults in diagnostic reasoning with diagnostic errors and patient harm. Acad Med 2012;87:149-56.

18 Schiff GD, Hasan O, Kim S, et al. Diagnostic error in medicine: analysis of 583 physician-reported errors. Arch Intern Med 2009;169:1881-7.

19 Newman-Toker DE, Schaffer AC, Yu-Moe CW, et al. Serious misdiagnosis-related harms in malpractice claims: The "Big Three" - vascular events, infections, and cancers. Diagnosis 2019;6:227-40.

20 Roadmap for Research to Improve Diagnosis. Society to improve diagnosis in medicine. Available: https://www. improvediagnosis.org/roadmap/ [Accessed 22 Jun 2020].

21 Singh H, Giardina TD, Meyer AN, et al. Types and origins of diagnostic errors in primary care settings. JAMA 2013;173:418-25.

22 Singh H, Sittig DF. Advancing the science of measurement of diagnostic errors in healthcare: the safer DX framework. BMJ Qual Saf 2015;24:103-10.

23 Schiff GD, Kim S, Abrams R, et al. Diagnosing diagnosis errors: lessons from a multi-institutional collaborative project, 2005. Available: https://www.ncbi.nlm.nih.gov/books/n/aps2/ A2551/?report $=$ reader [Accessed 7 May 2017].
24 Singh H, Daci K, Petersen LA, et al. Missed opportunities to initiate endoscopic evaluation for colorectal cancer diagnosis. Am J Gastroenterol 2009;104:2543-54.

25 Singh H, Hirani K, Kadiyala H, et al. Characteristics and predictors of missed opportunities in lung cancer diagnosis: an electronic health record-based study. J Clin Oncol 2010;28:3307-15.

26 Wahls TL, Peleg I. Patient- and system-related barriers for the earlier diagnosis of colorectal cancer. BMC Fam Pract 2009; 10:65.

27 Hussain F, Cooper A, Carson-Stevens A, et al. Diagnostic error in the emergency department: learning from national patient safety incident report analysis. BMC Emerg Med 2019;19:77.

28 Society to Improve Diagnosis in Medicine. DxQI seed grant program. Available: https://www.improvediagnosis.org/dxqi/ [Accessed 16 Jun 2020].

29 Singh H, Upadhyay DK, Torretti D. Developing health care organizations that Pursue learning and exploration of diagnostic excellence: an action plan. Academic Medicine 2020. [Epub ahead of print: 29 Oct 2019]. doi:10.1097/ ACM.0000000000003062.

30 Singh H, Bradford A, Goeschel C. Operational measurement of diagnostic safety: state of the science. Rockville, MD: Agency for Healthcare Research and Quality, 2020. https:// www.ahrq.gov/sites/default/files/wysiwyg/topics/state-ofscience

31 Croskerry P. The importance of cognitive errors in diagnosis and strategies to minimize them. Acad Med 2003;78:775-80.

32 Mamede S, de Carvalho-Filho MA, de Faria RMD, et al. 'Immunising' physicians against availability bias in diagnostic reasoning: a randomised controlled experiment. BMJ Qual Saf 2020;29:550-9.

33 Singh H, Zwaan L. Web Exclusives. Annals for Hospitalists Inpatient Notes - Reducing Diagnostic Error-A New Horizon of Opportunities for Hospital Medicine. Ann Intern Med 2016;165:HO2.

34 Braun LT, Zwaan L, Kiesewetter J, et al. Diagnostic errors by medical students: results of a prospective qualitative study. BMC Med Educ 2017;17:191. 